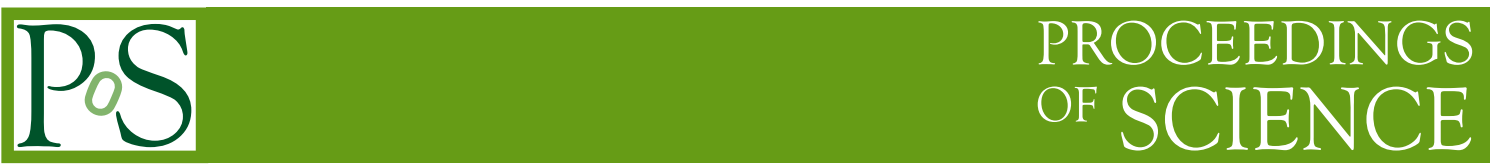

\title{
Charm physics in NA61/SHINE
}

\author{
Wojciech Brylinski ${ }^{* \dagger}$ \\ for the NA61/SHINE Collaboration \\ Warsaw University of Technology \\ E-mail: wojciech.brylinski@cern.ch
}

NA61/SHINE (SPS Heavy Ion and Neutrino Experiment) is a fixed-target experiment operating at the CERN SPS accelerator. The main goal of the Collaboration is to study the properties of the phase transition between confined matter and quark-gluon plasma by performing a two-dimensional scan of the phase diagram of strongly interacting matter. Within this program, collisions of different systems $(\mathrm{p}+\mathrm{p}, \mathrm{Be}+\mathrm{Be}, \mathrm{Ar}+\mathrm{Sc}, \mathrm{Xe}+\mathrm{La}, \mathrm{Pb}+\mathrm{Pb}$ ) over a wide range of beam momenta $(13 \mathrm{~A}-158 \mathrm{~A} \mathrm{GeV} / \mathrm{c})$ have been recorded.

Recently, the physics program of NA61/SHINE was extended by measurements of open charm production in A+A collisions which is the main goal of NA61/SHINE beyond 2020. In order to meet the challenges of the required spatial resolution of primary and secondary vertex reconstruction, the detector was upgraded by a micro vertex detector. A Small-Acceptance version of the Vertex Detector (SAVD) was successfully commissioned in December 2016 and first pilot data were collected for $\mathrm{Pb}+\mathrm{Pb}$ collisions at a beam momentum of $150 \mathrm{~A} \mathrm{GeV/c}$. This data allowed to validate the measurement concept and to perform the first direct measurement of open charm hadron production in collisions of nuclei at the SPS energies. In October and November 2017, large statistics datasets for $\mathrm{Xe}+\mathrm{La}$ collisions at $75 \mathrm{~A}$ and $150 \mathrm{~A} \mathrm{GeV} / \mathrm{c}$ were registered and are under analysis now. This contribution will present the motivation of open charm studies as well as the current status and details of the analysis of the collected $\mathrm{Pb}+\mathrm{Pb}$ and $\mathrm{Xe}+\mathrm{La}$ data.

International Conference on Hard and Electromagnetic Probes of High-Energy Nuclear Collisions 30 September - 5 October 2018

Aix-Les-Bains, Savoie, France

\footnotetext{
${ }^{*}$ Speaker.

${ }^{\dagger}$ This work was partially supported by the National Science Centre, Poland (grant 2015/18/M/ST2/00125).
} 


\section{Introduction}

SPS Heavy Ion and Neutrino Experiment (SHINE) [1] is a fixed-target experiment operating at the Super Proton Synchrotron (SPS) at the European Organization for Nuclear Research (CERN). The experiment is dedicated to explore the phase diagram of strongly interacting matter. Recently, the physics program of the NA61/SHINE experiment was extended by the measurement of open charm hadrons. It was motivated by three main questions:

- What is the mechanism of open charm production?

- How does the onset of deconfinement impact open charm production?

- How does the formation of quark-gluon plasma impact $J / \psi$ production?

In order to answer all of these questions, the mean multiplicity of charm quarks $\langle c \bar{c}\rangle$ produced in full phase space in heavy-ion collisions has to be known. Up to now, such data do not exist. The acceptance of the NA61/SHINE detector is large enough to extrapolate the measurements to the full phase space with relatively small uncertainties. This unique feature makes NA61/SHINE the only experiment which is able to perform such a measurement in the near future. Section 2 illustrates by examples the questions listed above.

\section{Motivation of open charm measurements}

The predictions of different models on the mean multiplicity of charm quark pairs $\langle c \bar{c}\rangle$ produced in central $\mathrm{Pb}+\mathrm{Pb}$ collisions at $158 \mathrm{~A} \mathrm{GeV} / \mathrm{c}$ are presented in Figure 1 (Left). The main conclusion from the plot is that the predictions differ by about two orders of magnitude. These very different models could coexist up to now because of the lack of the experimental data. Therefore, the precise measurement of $\langle c \bar{c}\rangle$ will narrow the spectrum of theoretical predictions and thus will allow to better understand the charm quark and hadron production mechanisms.

The second argument for the measurements of open charm production is related to the charm yield as a signal of deconfinement. Charm production in confined hadronic matter and in the quark-gluon plasma (QGP) is expected to be different due to the different carriers of charm in both phases. In the hadron gas, the lightest, most abundant charm carriers are D mesons, while in the QGP charm is carried by charm quarks. This is why the production of a pair of charm carriers in the confined state $\left(2 m_{\mathrm{D}} \approx 3.7 \mathrm{GeV}\right)$ requires an additional energy of about $1 \mathrm{GeV} / \mathrm{c}$ in comparison to the production of a pair of charm carriers in the QGP $\left(2 m_{c} \approx 2.6 \mathrm{GeV}\right)$. Thus, one expects to observe an enhancement of charm production when going from the confined state to the QGP. This can be considered as a signal of deconfinement.

Example model predictions of the Statistical Model of the Early Stage (SMES) [2] are presented in Figure 1 (Middle). The energy dependence of $\langle c \bar{c}\rangle$ is plotted. According to this model the enhancement of charm production is expected to be observed above an energy of about $\sqrt{\mathrm{s}_{\mathrm{NN}}}=$ $7-11 \mathrm{GeV}$. At $150 \mathrm{~A} \mathrm{GeV} / c\left(\sqrt{\mathrm{S}_{\mathrm{NN}}} \approx 16.8 \mathrm{GeV}\right)$ an enhancement by a factor of about 4 is predicted (when compared to the scenario without the phase transition).

The measurement of the mean multiplicity of charm quarks $\langle c \bar{c}\rangle$ will allow to verify the results predicted by the model and check whether the charm yield can be used as a signal of deconfinement. 

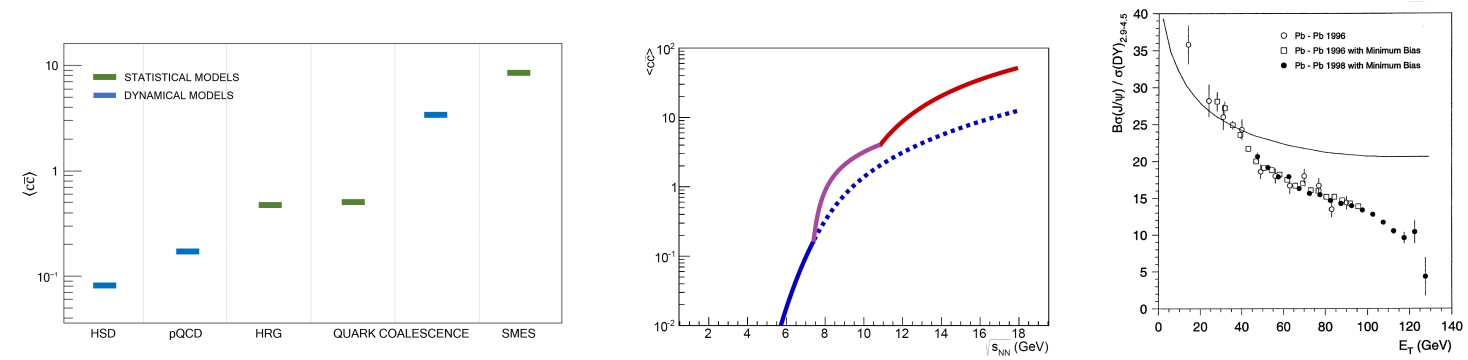

Figure 1: (Left) Mean multiplicities of charm quark pairs produced in full phase space in central $\mathrm{Pb}+\mathrm{Pb}$ collisions at $158 \mathrm{~A} \mathrm{GeV} / c$ calculated within statistical models (green bars) (the Hadron Resonance Gas model (HRG) [3], the Statistical Quark Coalescence model [3] and the Statistical Model of the Early Stage (SMES) [4]) as well as dynamical models (blue bars) (the Hadron String Dynamics (HSD) model [5], a pQCD-inspired model [6, 7] and the Dynamical Quark Coalescence model [8]). (Middle) Energy dependence $\left(\sqrt{S_{\mathrm{NN}}}\right.$ - center of mass energy per nucleon pair) of the mean multiplicity of charm quark pairs according to the SMES model [2]. Dotted (solid) line represents the scenario without (with) phase transition (the energy of the onset of deconfinement is approximately $\sqrt{\mathrm{S}_{\mathrm{NN}}} \approx 7 \mathrm{GeV}$ ). (Right) The branching ratio (B) multiplied $\sigma_{\mathrm{J} / \psi}$ divided by $\sigma_{\mathrm{DY}}$ as a function of transverse energy (measure of collision centrality) in $\mathrm{Pb}+\mathrm{Pb}$ collisions at $158 \mathrm{~A} \mathrm{GeV/c}$ measured by NA50 [10]. The curve represents the $\mathrm{J} / \psi$ suppression due to ordinary ("cold") nuclear matter absorption.

The last motivation point concerns $J / \psi$ suppression. It can be explained within the MatsuiSatz model [9] as a consequence of QGP formation. When the QGP is created, the strong interactions between produced $c$ and $\bar{c}$ quarks are screened by other quarks and gluons. Thus, the probability that $c$ and $\bar{c}$ quarks will bind decreases, reducing the probability of $J / \psi$ production.

The probability of $J / \psi$ production is given by the following formula: $P(c \bar{c} \rightarrow J / \psi) \equiv \frac{\langle J / \psi\rangle}{\langle c \bar{c}\rangle} \equiv$ $\frac{\sigma_{J / \psi}}{\sigma_{c \bar{c}}}$, where $\langle\ldots\rangle$ represents mean multiplicities and $\sigma$ the corresponding cross-sections. Thus, in order to calculate the probability of a $c \bar{c}$ pair hadronizing to $J / \psi$, data on the mean multiplicity of both $J / \psi$ and $c \bar{c}$ in full phase space are required. The $J / \psi$ yields were precisely measured by other SPS experiments, while $\langle c \bar{c}\rangle$ was not measured before. NA61/SHINE started such measurements in 2016.

In order to analyse the rich $J / \psi$ production measurements, it was up to now assumed that the mean multiplicity of $c \bar{c}$ quarks is proportional to the mean multiplicity of Drell-Yan pairs [9, 10]: $\langle c \bar{c}\rangle \sim\langle D Y\rangle$. Based on this assumption, the results of the NA50 experiment, presented in Figure 1 (Right), were interpreted as evidence of QGP formation in central $\mathrm{Pb}+\mathrm{Pb}$ collisions at $158 \mathrm{~A} \mathrm{GeV} / c$. The precise measurement of $\langle c \bar{c}\rangle$ will allow to verify this very important conclusion.

\section{NA61/SHINE detector}

The NA61/SHINE detector is a multi-purpose spectrometer optimised to study hadron production in different types of collisions: $\mathrm{p}+\mathrm{p}, \mathrm{h}+\mathrm{A}$ (hadron+nucleus), $\mathrm{A}+\mathrm{A}$ (nucleus+nucleus). A schematic picture of the detector is presented in Figure 2 (Left). The NA61/SHINE detector setup has excellent capabilities in charged particle momentum measurements and identification by a set of eight Time Projection Chambers (TPC) as well as Time-of-Flight (ToF) detectors. In 2016, the spectrometer was upgraded by adding Small-Acceptance Vertex Detector (SAVD). A photo of the 
SAVD is presented in Figure 2 (Right). The SAVD is built from 16 MIMOSA-26 silicon sensors located in four stations separated by $5 \mathrm{~cm}$ along the beam axis. According to simulation using A Multi-Phase Transport Model (AMPT) [11], the SAVD allows to reconstruct about 5\% of all $\mathrm{D}^{0}$ decays in the decay channel $D^{0} \rightarrow \pi^{+}+K^{-}$passing the analysis cuts.
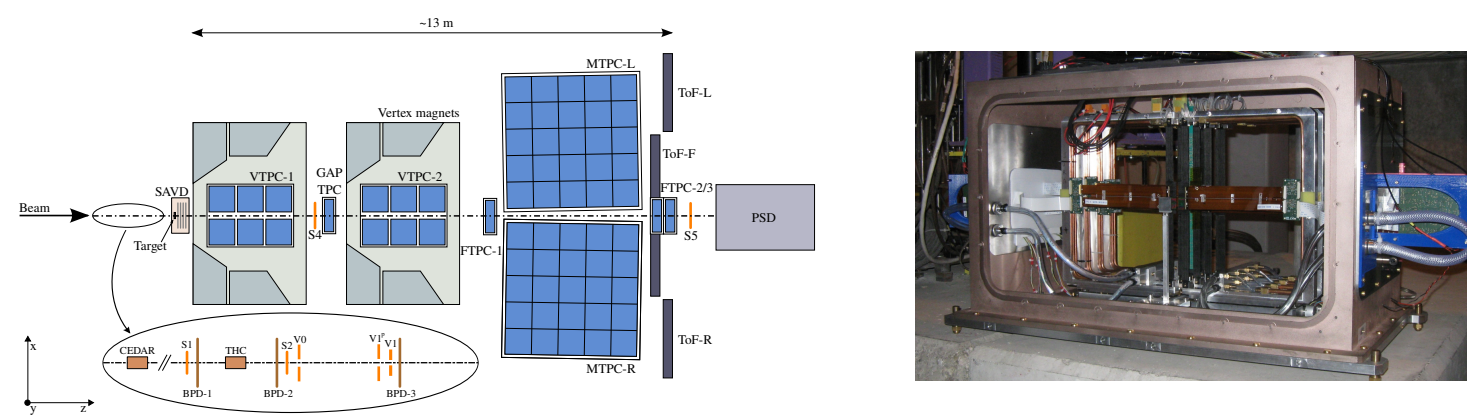

Figure 2: (Left) Schematic picture of the NA61/SHINE detector. (Right) Photo of the SAVD used by NA61/SHINE.

\section{SAVD performance}

The first test of the SAVD was performed in December 2016 in $\mathrm{Pb}+\mathrm{Pb}$ collisions at $150 \mathrm{~A}$ $\mathrm{GeV} / c$. The obtained primary vertex resolution along the beam direction of $30 \mu \mathrm{m}$ was sufficient to perform a search for the $\mathrm{D}^{0}$ and $\overline{\mathrm{D}^{0}}$ signals. Figure 3 (Left) shows the first indication of a $\mathrm{D}^{0}$ and $\overline{\mathrm{D}^{0}}$ peak obtained using the data collected during the $\mathrm{Pb}+\mathrm{Pb}$ run in 2016.

Successful performance of the SAVD in 2016 led to the decision to also use it during the $\mathrm{Xe}+\mathrm{La}$ data taking in 2017. About $5 \cdot 10^{6}$ events of central $\mathrm{Xe}+\mathrm{La}$ collisions at $150 \mathrm{~A} \mathrm{GeV} / \mathrm{c}$ were collected in October and November 2017. During these measurements the thresholds of the MIMOSA-26 sensors were tuned to obtain high hit detection efficiency which led to significant improvement in the primary vertex reconstruction precision. The spatial resolution of the primary vertices obtained for $\mathrm{Xe}+\mathrm{La}$ data is at the level of $1 \mu \mathrm{m}$ and $15 \mu \mathrm{m}$ in the transverse and longitudinal coordinates, respectively. The distribution of the longitudinal coordinate of the primary vertex is shown in Figure 3 (Right). The Xe+La data are currently under analysis and are expected to lead to physics results in the coming months.

The SAVD was also used during $\mathrm{Pb}+\mathrm{Pb}$ data taking at $150 \mathrm{~A} \mathrm{GeV/c}$ in November 2018 when about $4 \cdot 10^{6}$ events were collected.

\section{NA61/SHINE detector upgrades}

During the Long Shutdown 2 at CERN (2019-2020), the NA61/SHINE spectrometer is planned to be upgraded [12]. Most of the upgrades are necessitated by the charm physics program which requires the increase of the phase space coverage of the vertex detector and a tenfold increase of the data taking rate to about $1 \mathrm{kHz}$.

According to AMPT simulations, the new NA61/SHINE vertex detector will be able to reconstruct about $13 \%$ (about 3 times more than the SAVD) of all $\mathrm{D}^{0}$ decays in the decay channel: $D^{0} \rightarrow \pi^{+}+K^{-}$and about $9 \%$ of all $\mathrm{D}^{+}$decays in the decay channel: $D^{+} \rightarrow \pi^{+}+\pi^{+}+K^{-}$. 

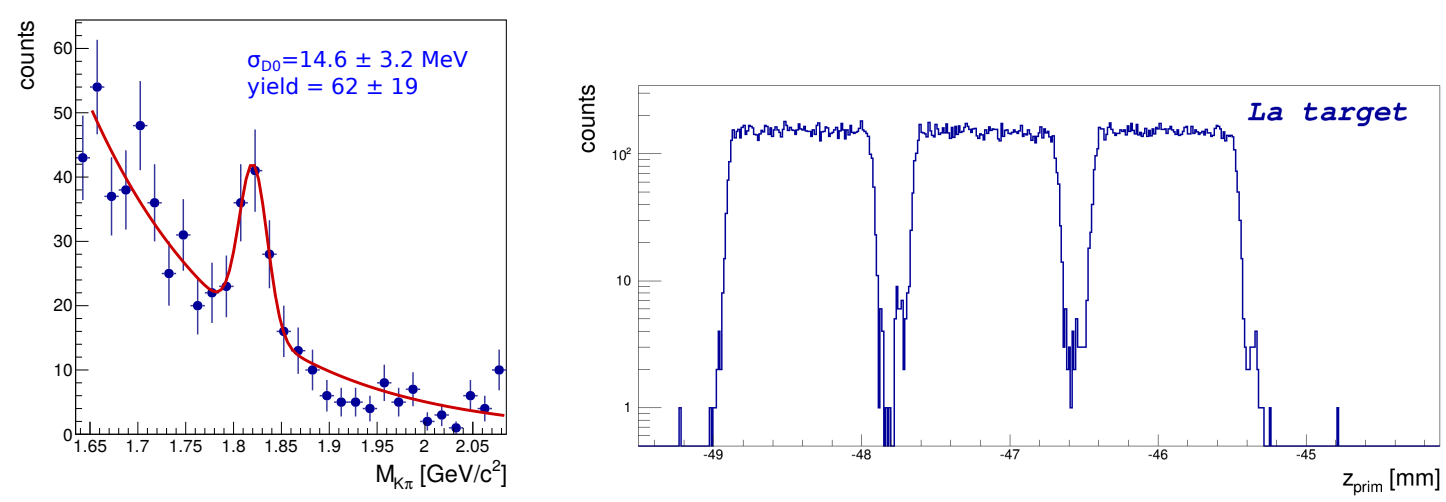

Figure 3: (Left) Invariant mass distribution of $\mathrm{D}^{0}$ and $\overline{\mathrm{D}^{0}}$ candidates in central $\mathrm{Pb}+\mathrm{Pb}$ collisions at $150 \mathrm{~A}$ $\mathrm{GeV} / \mathrm{c}$ after the background suppression cuts. (Right) Preliminary result from Xe+La collisions at $150 \mathrm{~A}$ $\mathrm{GeV} / c$ : distribution of longitudinal coordinate of the primary vertex for interactions in the La target, which was composed of three $1 \mathrm{~mm}$ plates.

\section{References}

[1] N. Abgrall et al. [NA61 Collaboration], JINST 9, P06005 (2014) doi:10.1088/1748-0221/9/06/P06005 [arXiv:1401.4699 [physics.ins-det]].

[2] R. V. Poberezhnyuk, M. Gazdzicki and M. I. Gorenstein, Acta Phys. Polon. B 48, 1461 (2017) doi:10.5506/APhysPolB.48.1461 [arXiv:1708.04491 [nucl-th]].

[3] A. P. Kostyuk, M. I. Gorenstein, H. Stoecker and W. Greiner, Phys. Lett. B 531, 195 (2002) doi:10.1016/S0370-2693(02)01488-0 [hep-ph/0110269].

[4] M. Gazdzicki and M. I. Gorenstein, Acta Phys. Polon. B 30, 2705 (1999) [hep-ph/9803462].

[5] O. Linnyk, E. L. Bratkovskaya and W. Cassing, Int. J. Mod. Phys. E 17, 1367 (2008) doi:10.1142/S0218301308010507 [arXiv:0808.1504 [nucl-th]].

[6] R. V. Gavai, S. Gupta, P. L. McGaughey, E. Quack, P. V. Ruuskanen, R. Vogt and X. N. Wang, Int. J. Mod. Phys. A 10, 2999 (1995) doi:10.1142/S0217751X95001431 [hep-ph/9411438].

[7] P. Braun-Munzinger and J. Stachel, Phys. Lett. B 490, 196 (2000) doi:10.1016/S0370-2693(00)00991-6 [nucl-th/0007059].

[8] P. Levai, T. S. Biro, P. Csizmadia, T. Csorgo and J. Zimanyi, J. Phys. G 27, 703 (2001) doi:10.1088/0954-3899/27/3/357 [nucl-th/0011023].

[9] T. Matsui and H. Satz, Phys. Lett. B 178, 416 (1986). doi:10.1016/0370-2693(86)91404-8

[10] M. C. Abreu et al. [NA50 Collaboration], Phys. Lett. B 477, 28 (2000). doi:10.1016/S0370-2693(00)00237-9

[11] Z. W. Lin, C. M. Ko, B. A. Li, B. Zhang and S. Pal, Phys. Rev. C 72, 064901 (2005) doi:10.1103/PhysRevC.72.064901 [nucl-th/0411110].

[12] A. Aduszkiewicz et al. [NA61 Collaboration], CERN-SPSC-2018-008, SPSC-P-330-ADD-10 